\title{
CONTROLLING SPATIAL CHAOS IN METAPOPULATIONS WITH LONG-RANGE DISPERSAL
}

\author{
- MICHAEL DOEBELI \\ Zoology Institute, \\ University of Basel, \\ CH-4051 Basel, Switzerland
}

(E.mail: doebeli@ubaclu.unibas.ch)

\author{
- GRAEME D. RUXTON \\ Division of Environmental and Evolutionary Biology, \\ University of Glasgow, \\ Glasgow G12 8QQ, United Kingdom \\ (E.mail: gdrlm@udcf.gla.ac.uk)
}

\begin{abstract}
We propose two methods to control spatial chaos in an ecological metapopulation model with long-range dispersal. The metapopulation model consists of local populations living in a patchily distributed habitat. The habitat patches are arranged in a one-dimensional array. In each generation, density-dependent reproduction occurs first in each patch. Then individuals disperse according to a Gaussian distribution. The model corresponds to a chain of coupled oscillators with long-range interactions. It exhibits chaos for a broad range of parameters. The proposed control methods are based on the method described by Güémez and Matías for single difference equations. The methods work by adjusting the local population sizes in a selected subset of all patches. In the first method (pulse control), the adjustments are made periodically at regular time intervals, and consist of always removing (or adding) a fixed proportion of the local populations. In the second method (wave control), the adjustments are made in every generation, but the proportion of the local population that is affected by the control changes sinusoidally. As long as dispersal distances are not too low, these perturbations can drive chaotic metapopulations to cyclic orbits whose period is a multiple of the control period. We discuss the influence of the magnitude of the pulses and wave amplitudes, and of the number and the distribution of controlled patches on the effectiveness of control. When the controls start to break down, interesting dynamic phenomena such as intermittent chaos can be observed. (C) 1997 Society for Mathematical Biology
\end{abstract}

1. Introduction. Chaos is a paradigm in ecology. It implies the possibility of very complicated population dynamics as a consequence of feedback mechanisms such as resource exploitation. Large, irregular, and unpredictable fluctuations in population size can occur even in the absence of 
external perturbations like those caused by changing climatic conditions. The quest for understanding how often and under which conditions complex dynamics occur in natural populations is a major driving force for contemporary ecological research.

One important line of thinking in this context concerns the inclusion of spatial structure into population dynamic models. It has been shown both empirically and theoretically that the dynamics of metapopulations (Levins, 1970) can be very different from those of their constituent local populations (for a review, see Gilpin and Hanski, 1991). Thus, spatial structure can prevent extinction (e.g. Huffaker, 1958; Hassell et al., 1991; Holt and Hassell, 1993), and it can affect the complexity of population dynamics. In particular, it has been argued that spatial extension often has a simplifying effect on population dynamics (McCallum, 1992; Hastings 1993; Gyllenberg et al., 1993; Stone, 1993; Ruxton, 1994; Doebeli, 1995a; Lloyd, 1995). On the other hand, Hastings and Higgins (1994) have shown that spatial structure can lead to new and interesting dynamic phenomena. They observed spatial chaos and extremely long transient behaviour in a metapopulation model with one-dimensional spatial extension and long-range dispersal. In this paper, we build on their model and combine it with another line of thought that is of increasing importance in chaos theory: the idea of chaos control.

In a seminal paper, Ott et al. (1990) showed how one can use the sensitive dependence on initial conditions to direct and control the trajectories of chaotic systems. Thus, making use of the attributes of chaos, one can stabilize otherwise unstable periodic orbits by adjusting system parameters. Because of the complicated and delicate geometry of chaotic attractors, very small adjustments are enough to achieve control. The method of Ott $e t$ al. (1990) has been successfully applied to a variety of models and experimental systems in physics (for a review, see Shinbrot et al., 1993) and in chemistry (e.g. Chakravarti et al., 1995). Some attempts have been made to apply it in ecological and evolutionary modeling (Doebeli 1993, 1995a, b).

A drawback of the method is that the adjustments in the parameters necessary at a given point in time require a rather detailed knowledge of the system and its dynamics. Therefore, the method is difficult to apply to systems with many degrees of freedom and high-dimensional chaotic attractors, even though there are some cases in which such systems can be controlled. In particular, despite some success (Sepulchre and Baboyantz, 1993; Aranson et al., 1994; Astakhov et al., 1995; Brayman et al., 1995), it is still a largely open problem how to control systems exhibiting spatial chaos.

Güémez and Matías (1993) have proposed a different method to control chaos. Their method is more robust than that of Ott et al. (1990) in the sense that it does not require detailed information about the system. They propose not to change parameters of a system, but instead to change the dynamic variable itself. The size of the adjustment is a fixed proportion of the dynamic variable, and the adjustment is made periodically with a 
certain period often equal to that of the stabilized periodic orbit to which the system will be driven. The method works well for simple systems (Güémez and Matías, 1993; Solé and Menéndez de la Prida, 1995). In this paper, we apply two variants of this method to the spatial population model of Hastings and Higgins (1994). Using numerical simulations, we show that the proposed methods work well to control spatial chaos in this metapopulation model. The model is of a quite general nature, and can be viewed as a prototype for models of large numbers of coupled oscillators with longrange interactions. Therefore, we think that our methods may prove to be useful for a variety of dynamical systems with spatial extension, many degrees of freedom, and high-dimensional chaotic attractors.

2. The Model. In this section, we first describe the metapopulation model that we use, and we then explain the two control mechanisms that we applied to this dynamic system.

2.1. Metapopulations with long-range dispersal. We envisage a metapopulation consisting of many local populations living in a patchily distributed habitat that extends along a one-dimensional ecosystem such as a sea coast, a lake shore, or the edge of a forest. Thus, we consider a one-dimensional array of local populations that we label $0, \ldots, n$. Each local population lives in an isolated patch in which it reproduces and from which individuals disperse to other patches in the array. We assume that reproduction is density dependent due to competition for resources. The model is set in discrete time: in each generation, there is first density-dependent reproduction in each patch, and then dispersal between patches. The local population densities after the dispersal phase are the densities at the start of the next generation.

To model density dependent reproduction, we use the difference equation

$$
\begin{aligned}
N_{t+1} & =G\left(N_{t}\right) \\
& =N_{t} \cdot f\left(N_{t}\right) \\
& =N_{t} \cdot \frac{\lambda}{1+\left(a N_{t}\right)^{b}}
\end{aligned}
$$

(Maynard Smith and Slatkin, 1973). This equation is among the most generally applicable one-dimensional ecological models (Bellows, 1981), and it is mathematically more flexible than the often used logistic and Ricker equations or than Hassell's (1975) equation (see Doebeli (1995a) for further discussion). In model (1), $N_{t}$ is the (local) population density at time $t$, and $f(N)$ is the density-dependent fitness function, i.e. the reproductive 
output per individual if the density in a patch is $N$. The parameter $\lambda$ is the intrinsic growth rate, and the parameter $b$ describes the type of competition that leads to density dependence. The parameter $a$ describes how well the individuals cope with the environment. For more details on the biological interpretation of these parameters, see Hassell (1975) and Schoener (1976). Equation (1) describes the dynamics of the local population in each of the patches of the metapopulation. For simplicity, we assume that all local populations have the same dynamics when isolated, i.e. that the demographic parameters $\lambda, b$ and $a$ are the same in all patches.

As is well known, the dynamics given by (1) can range from simple stable equilibria to chaos, depending on parameter values (e.g. May and Oster, 1976). Roughly speaking, the quantity that determines the dynamics of (1) is the derivative of the function $G$ at the equilibrium $N^{*}$, which is given by $f\left(N^{*}\right)=1$. This derivative is

$$
c=\frac{d G}{d N}\left(N^{*}\right)=1-b \frac{\lambda-1}{\lambda} .
$$

If $|c|<1$, then the equilibrium $N^{*}$ is locally stable. As $c$ decreases below -1 , the system exhibits the period-doubling route to chaos (May and Oster, 1976).

Let $N_{t}[i], i=0, \ldots, n$, be the local population density in patch $i$ at the start of generation $t$. The local population densities $M_{t}[i], i=0, \ldots, n$, after reproduction in generation $t$, but before dispersal are given by

$$
M_{t}[i]=G\left(N_{t}[i]\right) \quad i=0, \ldots, n .
$$

To complete the description of the dynamic metapopulation model, we have to describe dispersal between the local populations. Following Hastings and Higgins (1994), we assume that dispersal is given by a Gaussian distribution. After reproduction, a fixed proportion of the population in patch $i$ moves to patch $j$. This proportion is given by

$$
p(i, j)=\left(\frac{\pi}{D}\right)^{-1 / 2} \exp \left(-D(i-j)^{2}\right) .
$$

The parameter $D$ determines the dispersal range: a small $D$ implies large dispersal distances and vice versa. We note that $p(i, i)=(\pi / D)^{-1 / 2}$ is independent of $i$ and determines the fraction of each local population that remains in the patch.

Using (4), we can now determine the local populations after dispersal, i.e. at the beginning of the next generation. Given all the local populations densities $M_{t}[i]$ after reproduction in generation $t$, we sum up, for each patch $i$, the individuals arriving from the other patches $j=0, \ldots, n$ to 
obtain

$$
N_{t+1}[i]=\sum_{j=0}^{n} M_{t}[j] \cdot p(i, j) \quad i=0, \ldots, n
$$

Equations (3) and (5) describe the recursive relation between successive generations of the metapopulation model and therefore determine its dynamics.

Let us make a few remarks concerning this model. Equation (4) implies that dispersal is symmetric and that the patches are equally spaced with a distance of a unit of space between them. While symmetry and equidistance are clearly restrictive assumptions that are made here for simplicity, the size of the space unit does not matter, as any scaling can be compensated by changes in the parameter $D$. A further implicit assumption of the model is that the boundaries of the metapopulation are dissipative, that is, individuals that disperse to the left or the right of the array of patches representing the habitats of the metapopulation are lost. In the extreme case, only half of the individuals that disperse from the marginal populations living in patches 0 and $n$, respectively, reach another patch. The other half migrates from these marginal patches out of the system. This assumption seems realistic, and was also made by Hastings and Higgins (1994). An alternative, not explored here, would be to assume periodic boundary conditions, i.e. to assume that the patches 0 to $n$ are neighbouring patches, which would, for example, be realistic if the one-dimensional metapopulation would occupy the shore of a whole pond or lake.

On the other hand, equation (4) implies that no individuals are lost during dispersal except those leaving the metapopulation at the boundaries. This is an assumption that is commonly made in dispersal models describing coupled populations with discrete generations (e.g. Hastings, 1993; Gyllenberg et al., 1993; Doebeli, 1995a) but that might seem unrealistic. However, since we are assuming density-independent dispersal (which by itself is a questionable assumption), we can formally incorporate any losses due to dispersal within the metapopulation in the fitness function describing reproduction in each patch, e.g. by changing the intrinsic growth rate, and hence no generality is lost by this particular assumption.

We note that for fixed $i$, the sum over the $p(i, j)$, where $j$ runs from $-\infty$ to $+\infty$, is a discrete approximation to the integral over the Gaussian distribution with variance $1 / \sqrt{2 D}$, which is equal to 1 . Depending on $D$, this approximation might not be very accurate. Thus, for large $D$, it may happen that for some $i$, the sum $\sum_{j=0}^{n} p(i, j)$ is actually larger than 1 , which would mean that during dispersal, some individuals were added. To get around this problem, we normalized all the $p(i, j)$ by the expression $\Sigma=\sum_{j=0}^{n} p(n / 2, j)$. Thus, in our numerical simulations, we replaced all the $p(i, j)$ by $p(i, j) / \Sigma$. 
Finally, we remark that, although the model represented here is analogous to the model that Hastings and Higgins (1994) used for their numerical simulations, they actually had a different situation in mind in which space was continuous, that is, the habitat of the metapopulation was not patchy and organisms could thrive at any location in a continuous section of a sea coast. Hastings and Higgins (1994) used the space-discrete model described here for numerical approximation of their space-continuous model. However, the space-continuous model involves discrete-time population dynamics given by difference equations of the form (1) taking place on an infinitely small spatial area. We prefer the spatially discrete formulation of the metapopulation model, in which the local population dynamics given by (1) occur in discrete, finite sized patches of suitable habitat.

2.2. The control mechanisms. Güémez and Matías (1993) showed that the dynamics of difference equations of the form (1) describing the local populations in our model can be controlled by changing the dynamic variable $N$, here the population size, in the following way. Let $p$ be some positive integer; $p$ is the period of the control. Then in all generations that are multiples of $p$, we change the population size at the beginning of generation $t, N_{t}$, by a fixed proportion. More precisely, let $-1<\gamma<1$ be some real number in the interval $(-1,1)$. Then, at the beginning of generation $t$, we replace $N_{t}$ by $N_{t}(1+\gamma)$ if $t$ is a multiple of $p$. Thus, the control is given by

$$
\begin{aligned}
N_{t+1} & =G\left(N_{t}\right) \quad \text { if } p \text { does not divide } t \\
& =G\left(N_{t}(1+\gamma)\right) \quad \text { if } p \text { divides } t .
\end{aligned}
$$

Güémez and Matías (1993) showed that if the modulus of $\gamma$ is not too small and if $p$ is not too large, then this control mechanism typically forces the dynamics of the system on a pcriodic orbit with a period that is a multiple of $p$ (often the multiplication factor is 1; Güémez and Matías (1993) actually showed this for the logistic and for the Ricker equations, but their results are also true for other one-dimensional models such as the one considered here).

For our metapopulation model, we generalize this procedure as follows. Let $H=\{0, \ldots, n\}$ be the collection of patches in the metapopulation, and let $K \subset H$ be some subset of the patches. $K$ will be the time-invariant set of patches to which the control is applied. Thus, let $\gamma$ and $p$ be as above, that is, $\gamma$ determines the size of the control and $p$ its period. Then, in generation $t$, we replace the local population $N_{t}[i]$ in patch $i$ by $N_{t}[i](1+\gamma)$ if $t$ is a multiple of $p$, and if $i \in K$, that is, if the patch $i$ lies in the subset of patches $K$ chosen for control. Thus, in the controlled system, equations 
(3) are replaced by

$$
\begin{aligned}
& M_{t}[i]=G\left(N_{t}[i]\right) \quad i=0, \ldots, n \text { if } p \text { does not divide } t \\
& M_{t}[i]=\left\{\begin{array}{ll}
G\left(N_{t}[i]\right) & \text { if } i \notin K \\
G\left(N_{t}[i](1+\gamma)\right) & \text { if } i \in K
\end{array} \text { if } p \text { divides } t .\right.
\end{aligned}
$$

Thus, the method consists of a "pulse control," i.e. of periodically removing or adding (depending on whether $\gamma$ is negative or positive) a fixed proportion of the local populations in a fixed subset of all patches at the beginning of a generation, i.e. before reproduction. After reproduction, dispersal occurs as described in the previous subsection.

A variant of this control mechanism is obtained by replacing the pulse control by a "wave control." Here, the periodic pulses of fixed size to the patches in $K$ are replaced by a "controlling wave," i.e. by continual, sinusoidally varying controls: the wave control is applied in every generation, but the size and the direction of the control pulses vary. Formally, this control is described by

$$
M_{t}[i]= \begin{cases}G\left(N_{t}[i]\right) & \text { if } i \notin K \\ G\left(N_{t}[i]\left\{1+\gamma \cdot \cos \left(\frac{2 \pi t}{p}\right)\right\}\right) & \text { if } i \in K .\end{cases}
$$

Here, $\gamma$ is the amplitude of the controlling wave and $p$ is its period. Sinusoidally varying controls have previously been applied to eliminate chaos (Lima and Pettini 1993). However, as in the method of Ott et al. (1990), the controls were applied to parameters of the system, not to the dynamic variables themselves, and the controlled systems were of low dimension. Here, we will see that pulse and wave control of dynamic variables in a high-dimensional system work well to tame spatial chaos.

3. Results. Hastings and Higgins (1994) showed that the metapopulation model described in the previous section can have very complicated dynamics for some set of parameters. In particular, they showed that there may be extremely long transients before the system finally settles down on an attractor. When moving on these transients, the system experiences different dynamic regimes with abrupt changes between them, ranging from cyclic or quasi-periodic motion to spatial chaos. Here, we concentrate on parameter regions where these phenomena do not occur. In order to test our control mechanisms, we consider parameter regions where the system quickly settles on a complicated attractor leading to spatial choice. This can 
be achieved by choosing the parameters in equation (1) describing the local population dynamics in such a way that the complexity $|c|$, equation (2), of this difference equation is large enough. That there is spatial chaos without long transients can then be checked numerically (cf. Fig. 1A).

All results were obtained from numerical simulations. Figure 1 shows results of applying the first control mechanism described in the last section, i.e. the pulse control, to a chaotic metapopulation. Figure 1A shows the chaotic dynamics of an uncontrolled system with 80 local populations. In Fig. 1B, C, results of applying the pulse control with two different periods are shown. In these diagrams, the control starts after 1000 generations, that is, after 1000 time steps equations (3) are replaced by equations (7) in the iterative procedure. The set $K$ of controlled patches was chosen by including each patch with a probability of $20 \%$ using uniform random numbers. Thus, in (roughly) $20 \%$ of the patches, the control was applied, and the controlled patches were more or less evenly distributed over the whole metapopulation.

If the control period is low (Fig. 1B), the system is stabilized on a cyclic orbit with the same period as used in the control. If the control period gets larger (Fig. 1C), the control becomes less powerful and more imprecise: the system is still stabilized on a periodic orbit, but its period is twice the control period. In addition, it may take several hundred generations until the control becomes effective. These observations are consistent with the results of Güémez and Matías (1993) for simple models.

The effectiveness of the control also depends on the magnitude of the pulse, i.e. on the magnitude of $|\gamma|$, and on the proportion of controlled patches. This is illustrated in Fig. 2. In Fig. 2A, the period of the pulse control is the same as in Fig. 1B, but now the magnitude of $\gamma$ is too small for permanent control. However, the control almost works: for intermittent periods of time, the system moves on a trajectory that is almost a 2-cycle, but the systems always returns eventually, and abruptly, to highly chaotic fluctuations, after which it is again confined to simple dynamics for some time. The result is a rare example of spatial intermittent chaos. In simple systems, intermittent chaos (Pomeau and Manneville 1980) has previously been observed by Doebeli (1993, 1994, 1995a, b) and by Gavrilets and Hastings (1995) in ecological and evolutionary contexts. It implies radical and continual changes between different dynamic regimes.

In Fig. 2B, the period of the control and the value of $\gamma$ are the same as in Fig. 1B, but the proportion of controlled patches is smaller. Here, the control is also not able to confine the system to a periodic orbit. Instead, the dynamics remain chaotic, but the control has the effect of inducing much smaller fluctuations in the system. Figure $2 \mathrm{C}$ illustrates that in this case, too, the control "almost works." It shows the densities of the local populations in consecutive generations. Most of these local populations move on nearly cyclic orbits, except some of the marginal ones. For these, 


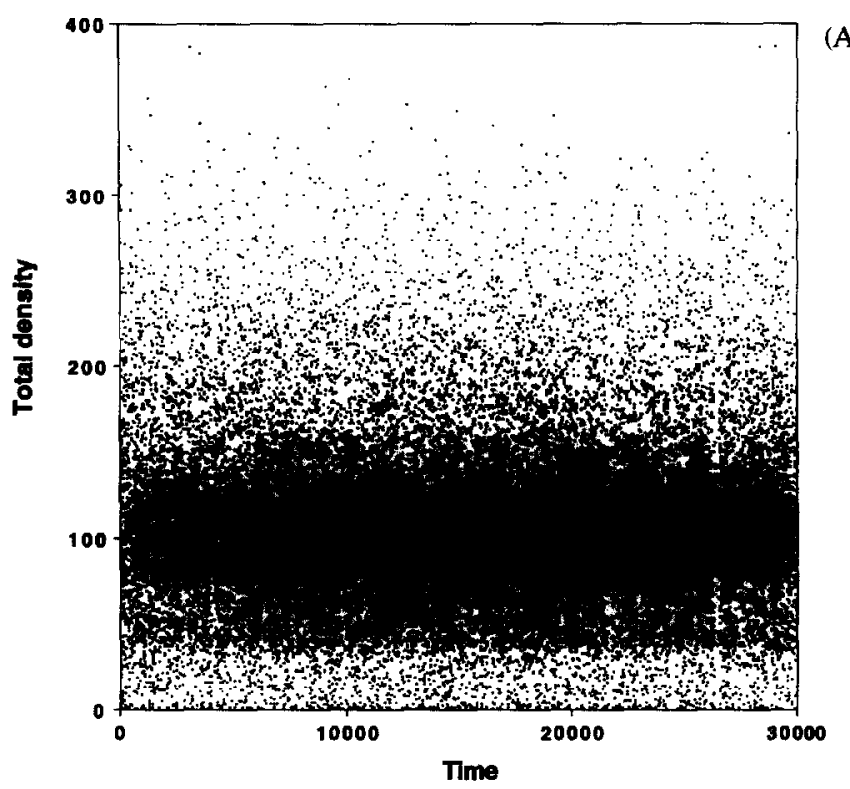

(A)

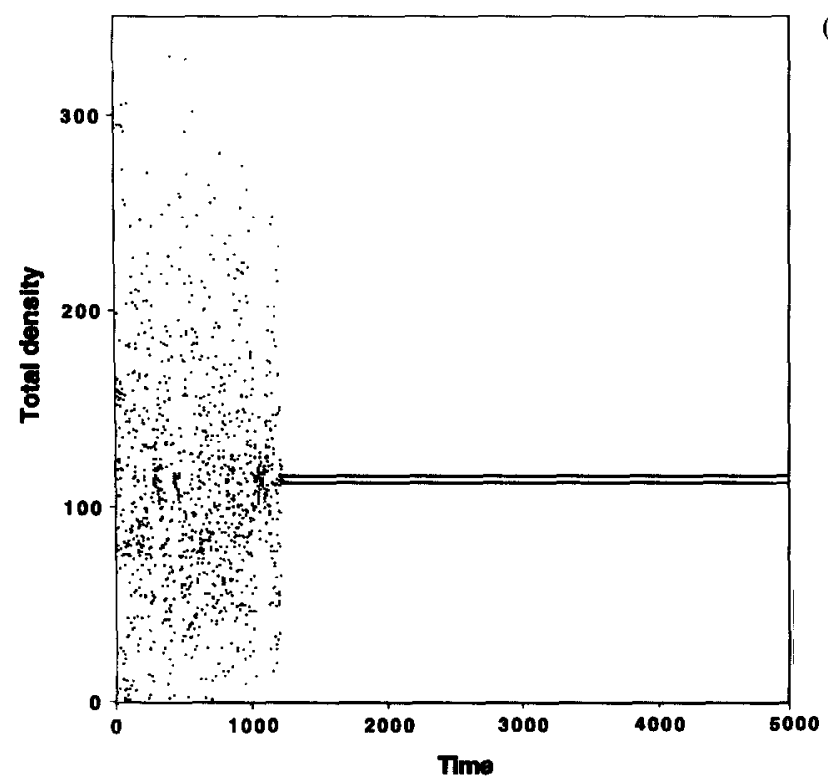

(B)

Figure 1. Effects of pulse control on a chaotic metapopulation. The time series of the total density of the uncontrolled metapopulation consisting of 80 local populations is shown in A for 30,000 generations to make sure that there is no transient behaviour. In $B$, the pulse control is applied to this metapopulation starting after 1000 generations with a control period of 2 . The system settles on a 2-cycle. The control was applied to 17 uniformly randomly chosen patches ( $\sim 20 \%$ of the 80 patches) and the magnitude of the control was $\gamma=-0.2$. For higher control periods, larger pulses and a large proportion of controlled patches are necessary for control. In $\mathrm{C}$, the system settles on a 10-cycle after a period 5 pulse control is applied with $\gamma=-0.4$ and 32 randomly chosen patches $(\sim 40 \%)$. It took ca. 300 time steps until the control was effective. Similar results would be obtained if the values of $\gamma$ were positive with the same modulus. The demographic parameters of the local populations given by equation (1) were: $\lambda=14, b=7.5$ and $a=1.4217$. The dispersal constant in equation (4) was $D=0.0111$. 


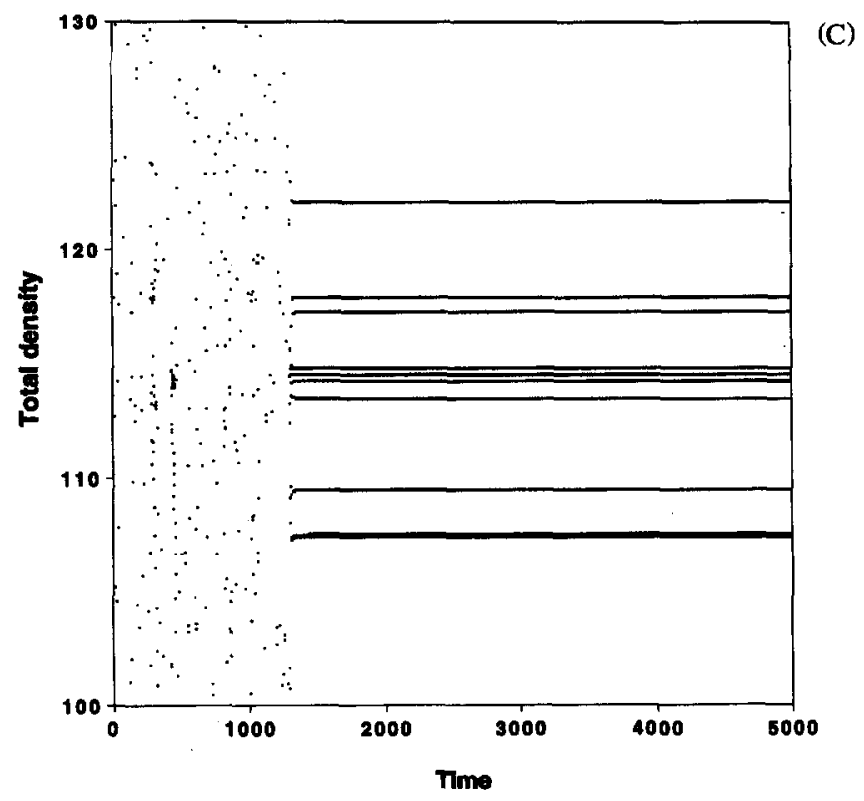

Figure 1. (Continued).

the control does not work, and they exhibit more irregular fluctuations. These "loose ends" prevent the whole metapopulation from moving on an exactly periodic orbit instead of merely exhibiting smaller fluctuations.

The fact that it is only a certain subset of the local populations which the control fails to confine to regular behaviour raises an interesting question. To which of the local patches should the control be applied? In other words, how should the subset $K$ be chosen? So far, we have worked with $K$ consisting of randomly chosen and evenly distributed local populations consisting of a certain proportion of all patches in the metapopulation. Perhaps surprisingly, the control method fails if this proportion is too high. For example, if instead of $20 \%$ as in Fig. 1B we chose $70 \%$ evenly distributed patches, there would be no visible effect of the control. Thus, the proportion of controlled patches must be low enough, e.g. to achieve control in Fig. 1B it must be lower than ca. 50\%.

Generalizing from simple metapopulations consisting of two patches (Gyllenberg et al., 1993), one expects that high dispersal distances, i.e. low values of $D$, would lead to some sort of synchronization in the metapopulation model (5), and hence to a reduction of its dimensionality. In this case, one could imagine that controlling all the patches would lead to simple dynamics. However, in the course of many numerical investigations, we have never found circumstances in which modifying all the patches in a large system lead to control of chaos. Nevertheless, in the absence of analytic proof, our conclusion that such action never leads to control should 


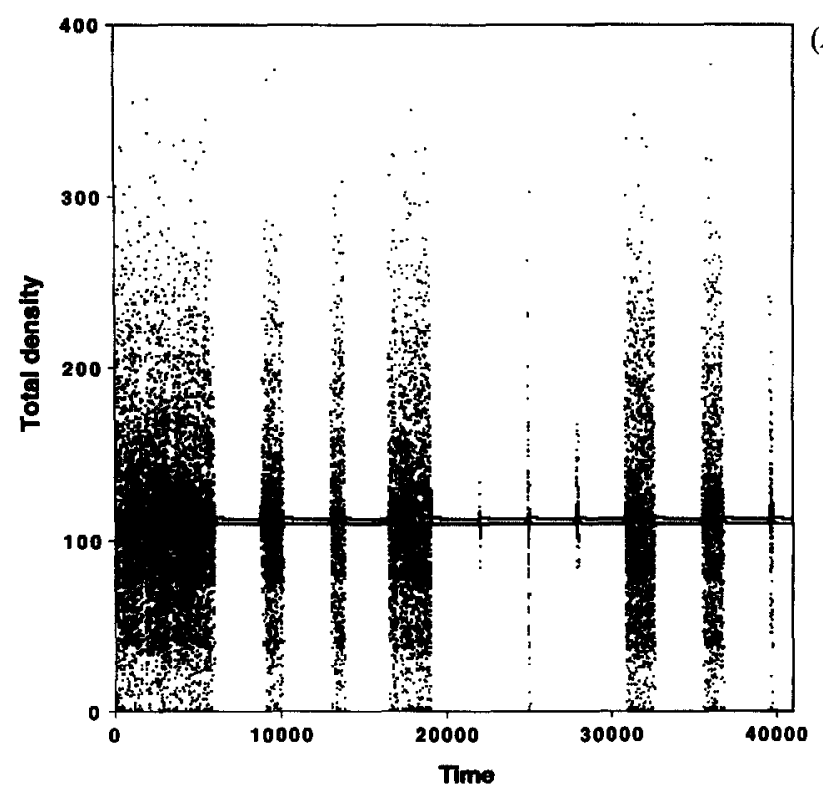

(A)

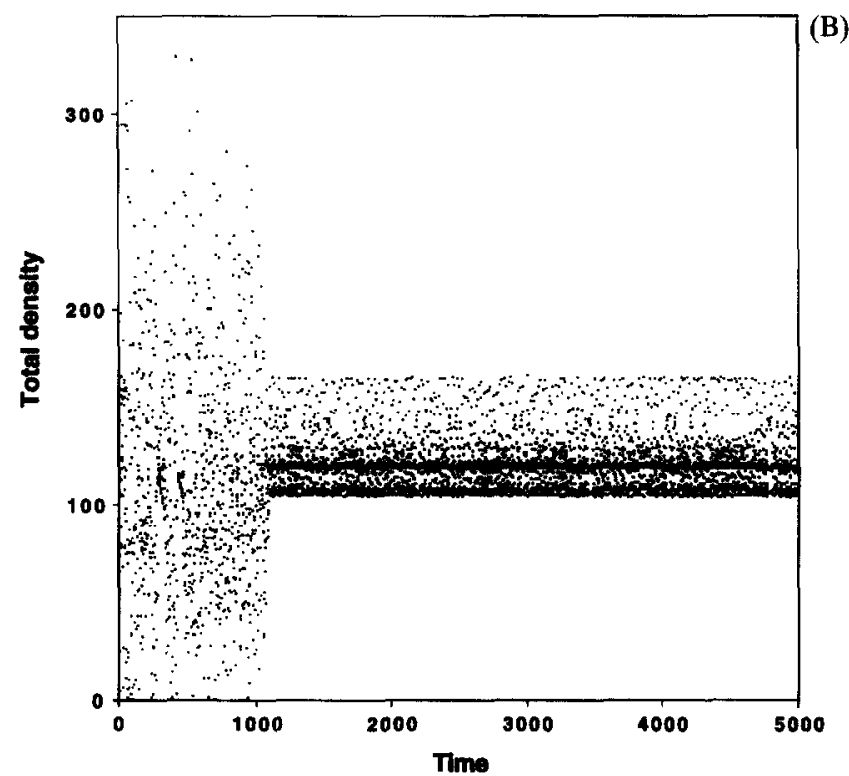

Figure 2. Effects of changing control parameters. The same metapopulation was used as in Figure 1. A is the same as Fig. 1B, except that the magnitude of the pulse is lower, $\gamma=-0.09679$. The control works for intermittent periods of time, which alternate with periods of chaotic fluctuations with abrupt changes between these dynamic regimes. $B$ is again the same as Fig. 1B, but the number of controlled patches is lower: 4 of the 17 patches uscd for Fig. 1B were left uncontrolled. The control is less effective, but still has the effect of inducing smaller fluctuations in the system. C shows what happens to the local populations in this scenario. The population densities in all 80 patches are shown in the eight successive generations following generation 5000 , i.e. the end of the simulation in B (in the plot, lines join the different local densities in each generation). Most local populations are nearly 2 -cyclic, except the marginal ones on the right. 


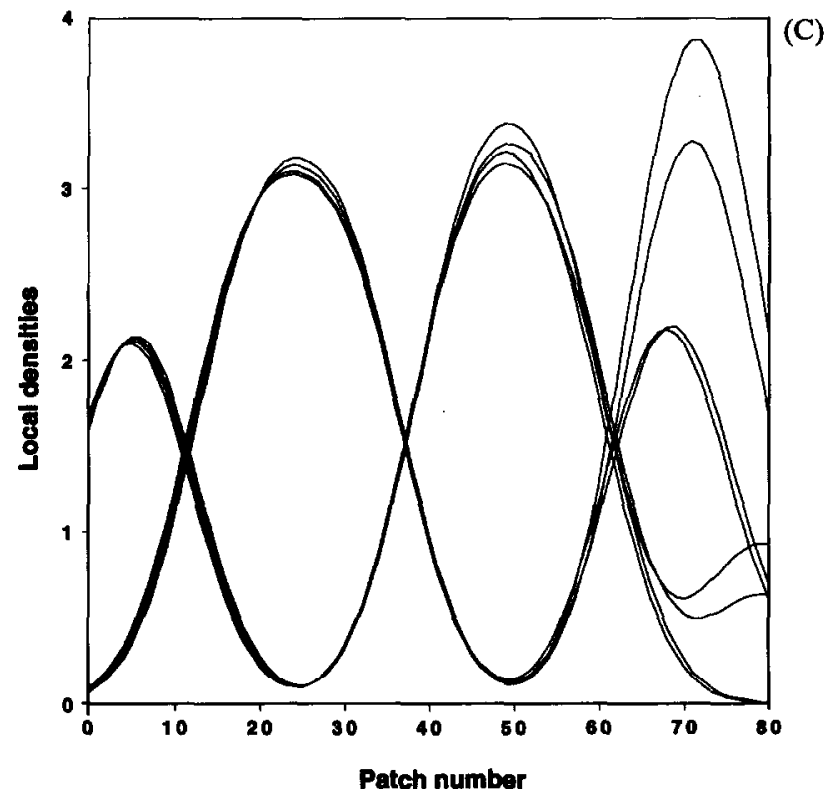

Figure 2. (Continued).

be tempered. The referee has provided us with an example of a very simple system consisting of three patches in which control can be achieved by modifying all three local populations. We hope that others will be able to clarify the generality of our observations.

Even if the proportion of controlled patches is low enough, it depends on their distribution whether the control mechanism has an effect or not. In general, control is harder to achieve if the controlled patches are clumped and away from the margins of the metapopulation. For example, if the $20 \%$ of controlled patches in Fig. 1B were chosen, instead of being evenly distributed, to be the central patches $42, \ldots, 57$ of the metapopulation, then the control would fail. Thus, a low enough proportion of controlled patches as well as an appropriate distribution of these patches are necessary conditions for an effective control.

However, as illustrated in Fig. 2B, and as is intuitively expected, control is also lost if the proportion of controlled patches is very low. In this case, the distribution of the controlled patches becomes even more important. For example, in Fig. 2B, the distribution of the relatively few (13) controlled patches is too even to achieve control. However, if, instead, the controlled patches are chosen to be the ones with numbers $0,10,20,30$ plus the six patches on the right margin of the metapopulation (i.e. numbers 75-80), then control is again possible. Even though now even fewer patches (ten) are controlled, the system settles on 4-cycle (not shown). This is because of 
the "loose ends" which cause the failure of control with evenly distributed control patches as described earlier: the "loose ends" effect disappears if the control is specifically applied to marginal patches (at least on one side).

All the results described so far also hold qualitatively for the second control mechanism described in section 2 , i.e. for the wave control given by equation (8). Figures 3 and 4 summarize some of the effects of wave
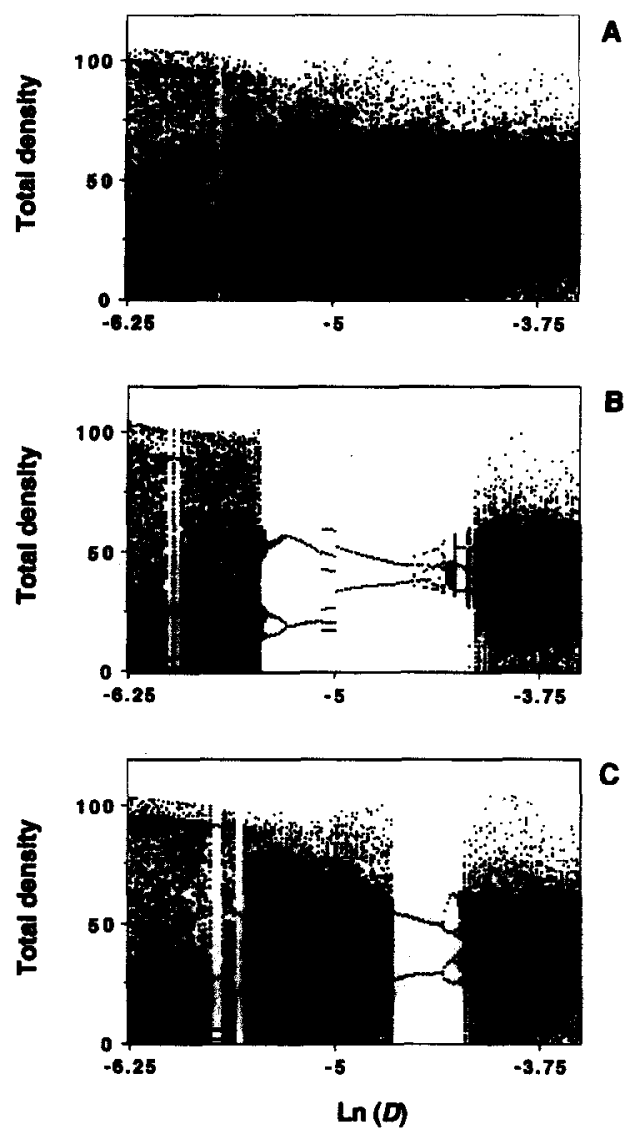

Figure 3. Bifurcation diagrams for the wave control. The logarithm of the dispersal parameter $D$ was used as bifurcation parameter. Small values of $\ln (D)$ correspond to large dispersal distances. A shows the uncontrolled dynamics of the total density of a metapopulation consisting of 41 local populations (i.e. $n=40$ ). In $B$, a period 2 wave control with $\gamma=-0.2$ was applied to the marginal patches $0-11$ and $29-40$ in the metapopulation, and in $C$, the same control was applied to the marginal patches $0-7$ and 33-40 and to central patches 16-24. The windows of regular dynamics are larger in the former case. The demographic parameters of the local populations were $\lambda=5, b=8.75$, and $a=1.172$. For 200 equally spaced values of $\ln (D)$, the metapopulation was first run without control for 500 time steps, then the control was applied for 700 time steps (no control in A). During the last 200 steps, the density was plotted. Selected numerical simulations indicated that long transients do not occur for the range of parameters considered, so that this procedure is appropriate. 

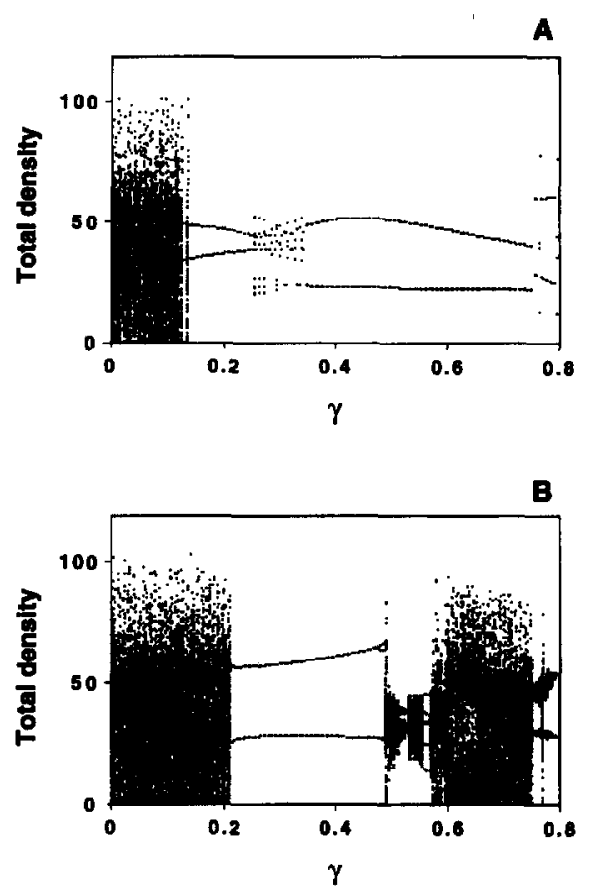

Figure 4. Bifurcation diagrams for the wave control. The control magnitude $\gamma$ was used as bifurcation parameter for the same metapopulation as in Fig. 3. In $\mathrm{A}, \mathrm{B}$, the dispersal parameter was $D=0.0136$, and in $\mathrm{C}, \mathrm{D}$, we set $D=0.0091$. In A, C, the marginal patches $0-11$ and $29-40$ were controlled, while in $B, D$, the control was applied to the marginal patches $0-7$ and 33-40 and to the central patches 16-24. The control works better when a larger proportion of marginal patches are controlled. The same procedure as for Fig. 3 was applied to obtain the diagrams.

control. In Fig. 3, bifurcation diagrams are shown with the parameter $D$ determining the dispersal distance as bifurcation parameter. Compared to the uncontrolled dynamics shown in Fig. 3A, the control mechanism induces windows of regular dynamics for intermediate dispersal distances. The control is least effective for very large and very low dispersal ranges. Similar results hold for the pulse control method. While for large dispersal distances control can often still be achieved if the control magnitude $\gamma$ is big enough, control is impossible for very small dispersal distances. In these cases, our numerical simulations suggest that not even high values of $\gamma$ are sufficient for control. For example, using the same demographic parameters as in Fig. 1 for the local populations but choosing $D$ so that the chance of dispersing over more than three neighbouring patches is less than $0.5 \%$, we were unable to find combinations of $\gamma, p$, and $K$ for either control mechanism which lead to a change in the chaotic dynamics exhibited by such a metapopulation. Thus, it seems that the control methods proposed 

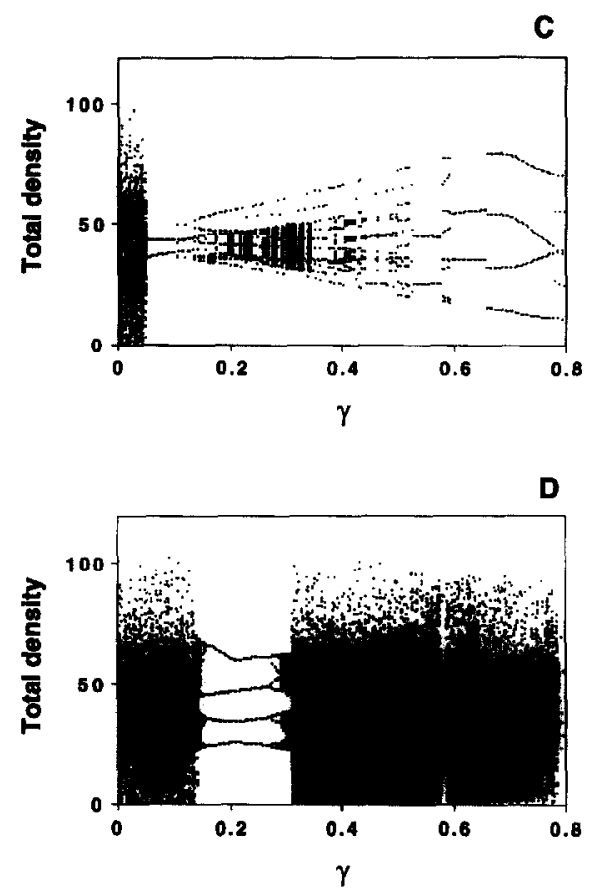

Figure 4. (Continued).

here only work for systems that are sufficiently connected, i.e. in which interactions between local populations occur over a sufficiently wide range.

The windows of regular dynamics induced by the control are larger if the control is applied only to marginal patches (Fig. 3B) than when it is applied also to patches lying in the center of the metapopulation (Fig. 3C). This reiterates what was said earlier about the influence of the distribution of the controlled patches on the effectiveness of the control. The phenomenon is again apparent in Fig. 4, where the control magnitude $\gamma$ was used as bifurcation parameter. If only marginal patches are used for the control, it works for larger ranges and for lower values of $\gamma$ than if the control is also applied to central patches. In addition, control altogether ceases to be effective in the scenarios considered in Figs. 3 and 4 if too many central patches are used for control. For example, even very high control magnitudes $\gamma$ are ineffective if the control is applied to the central patches 8-32, and a corresponding bifurcation diagram for the dispersal distance would be very similar to the one shown in Fig. 3A for an uncontrolled metapopulation.

Based on extensive numerical work, we believe that the illustrative examples of this section show general features of the behaviour of our model. However, we are unable to derive any analytical results, and so must urge some caution in interpreting our results until further numerical or 
analytical work is forthcoming. We hope that the results presented here will inspire others to carry out some of these extensions.

4. Discussion. The influence of spatial structure on ecological dynamics has become a focus of interest in recent years. Spatial heterogeneity can have profound effects on extinction probabilities and on the complexity of population dynamics. While many researchers have argued that dispersal between local populations can simplify population dynamics (McCallum, 1992; Hastings, 1993; Gyllenberg, 1993; Stone, 1993; Ruxton, 1994; Doebeli, 1995a; Lloyd, 1995), the addition of spatial extension increases the dimensionality of population dynamic models, which by itself may lead to new levels of complexity, as exemplified by the phenomenon of spatial chaos (Chow and Mallet-Paret 1995).

When faced with complexity, one can try to use the very properties of chaos to control the dynamics and elicit different types of regular behaviour that are present "behind the scenes" set by the chaotic attractor (Shinbrot et al., 1993). The most widely used method to control chaos is that proposed by Ott et al. (1990). It works by slightly adjusting system parameters according to a rule which has to be extracted from information about the dynamics of the system. For this reason, the method is difficult to apply in systems with many degrees of freedom and high-dimensional chaotic attractors. In particular, it is not clear how to apply this method to control spatial chaos. Some progress has been made (Sepulchre and Baboyantz, 1993; Aranson et al., 1994; Astakhov et al., 1995; Brayman et al., 1995), but chaos in chains of coupled difference equations of the type considered in this paper was only controlled for systems with very low spatial connectance, i.e. with a very small range of interactions between the spatially separated oscillators (Astakhov et al., 1995).

Here, we proposed a different method to control chaos in metapopulations with long-range dispersal. Our method is a generalization of the control mechanism proposed by Guiémez and Matías (1993) for single difference equations. The method has previously been applied to systems of coupled equations by Solé and Menéndez de la Prida (1995), but in a more restrictive way (only one equation was controlled), and in systems with much lower dimensions than the ones considered here.

The method of Güémez and Matías (1993) differs from that of Ott et al. (1990) in that the adjustments are made to the dynamic variables themselves (the population densities in our case) rather than to system parameters. However, this is not the main difference, as variants of the method of Ott et al. (1990) also used adjustments to dynamic variables (Lima and Pettini, 1993). The main difference is that in the Güémez and Matías (1993) method, the adjustments are made rather indiscriminately and without detailed information about the dynamics of the system. 
Thus, in the pulse control method that we applied to the metapopulation model described in section 2 , we changed the local population size in a subset of all patches by a fixed proportion and at regular time intervals. In the wave control method, local population sizes are changed in every generation, but the magnitude of the adjustment varies according to a sinusoidal curve. Neither method requires knowledge about the dynamics of the system, and both methods work well over a wide range of parameters.

The effectiveness of the control methods depends on the size of the adjustments as well as on the proportion and distribution of the controlled patches in the metapopulation. Large pulses or wave amplitudes facilitate control, and the distribution of the control patches should not be too clumped in only one region of the metapopulation. The proportion of controlled patches should neither be too low nor, somewhat surprisingly, too high. In the transitional regions where control ceases to be effective, interesting dynamic phenomena such as intermittent chaos can be observed (Fig. 2A).

The control mechanisms only work well in metapopulations in which dispersal distances are not very low. That is, they only work well if the connectance of the system is relatively high, and the interactions between local populations occur over a sufficiently wide range. Since the method of Ott et al. (1990) has been successfully applied to systems of coupled oscillators with interactions only between nearest neighbours, hence with a very low connectance (Asthakov et al., 1995), our theory therefore seems to neatly fill a gap in the theory of controlling spatial chaos.

Of course, an important question is: How feasible is it to apply these methods to natural systems? It does not seem too unrealistic ecologically to change local population sizes in the way required, particularly for the pulse control. In fact, because the method does not require a detailed knowledge of the dynamics of the system but only an assessment of the current (local) population size, it is tempting to speculate that metapopulations with chaotic fluctuations can be controlled by altering population sizes in selectively chosen habitat patches. For control with a negative pulse $\gamma$, a system manager would have to periodically harvest a fixed proportion of the local population in selected habitats. For positive $\gamma$, pulse control would correspond to periodically adding individuals to local populations. This could be achieved if a system manager had an independent reservoir of individuals at his disposal. Regarding the wave control, an interesting possibility opens up: if local populations are disturbed cyclically by external factors such as seasonal changes in the climate in some areas of a metapopulation, but not in others, then this might lead from otherwise chaotic dynamics to more regular dynamics with smaller fluctuations. Possibly this applies to species which have a spatially extended range longitudinally, the part closest to the equator being less affected by seasonal fluctuations than the part closer to 
the poles. Thus, natural wave control might be involved in controlling the dynamics of real populations and, to keep on speculating, it might be a reason why chaotic dynamics are rare in natural populations (I. Suárez, personal communication).

We hope that the control methods proposed here will open up new perspectives on controlling spatial chaos in other areas as well, e.g. in physics, or in neurology, where high-dimensional systems of coupled oscillators can be used to study brain dynamics, and where ideas of chaos control as a cognitive process have entered the literature (Sepulchre and Baboyantz, 1993; Solé and Menéndez de la Prida, 1995). The metapopulation model we used is sufficiently general to have potential applications in these fields. However, for both the metapopulation model of Hastings and Higgins (1994) and for our control mechanisms, it remains to be seen how they behave when the spatial dimension is larger than 1 .

We thank Mats Gyllenberg for careful reading of the paper and for many helpful suggestions.

\section{REFERENCES}

Aranson, I., H. Levine and L. Tsimring. 1994. Controlling spatiotemporal chaos. Phys. Reu. Lett. 72, 2561-2564.

Astakhov, V. V., V. S. Ansihchenko and A. V. Shabunin. 1995. Controlling spatiotemporal chaos in a chain of coupled logistic maps. IEEE Trans. Circuits Syst. I 42, 352-357.

Bellows, T. S., Jr. 1981. The descriptive properties of some models for density dependence. J. Anim. Ecol. 50, 139-156.

Brayman, Y., J. F. Lindner and W. L. Ditto. 1995. Taming spatiotemporal chaos with disorder. Nature 378, 465-467.

Chakravarti, S., M. Marek and W. H. Ray. 1995. Reaction-diffusion system with Brusselar kinetics - control of a quasiperiodic route to chaos. Phys. Rev. E 52, 2407-2423.

Chow, S. N. and J. Mallet-Paret. 1995. Pattern-formation and spatial chaos in lattice dynamical systems. IEEE Trans. Circuits Syst. I 42, 746-751.

Doebeli, M. 1993. The evolutionary advantage of controlled chaos. Proc. Roy. Soc. London $B$ 254, 281-286.

Doebeli, M. 1994. Intermittent chaos in population dynamics. J. Theor. Biol. 166, 325-330.

Doebeli, M. 1995a. Dispersal and dynamics. Theor. Pop. Biol. 47, 82-106.

Doebeli, M. 1995b. Updating Gillespie with controlled chaos. Amer. Nat. 146, 479-487.

Gavrilets, S. and A. Hastings. 1995. Intermittency and transient chaos from simple frequency-dependent selection. Proc. Roy. Soc. London B 261, 233-238.

Gilpin, M. and I. Hanski (Eds). 1991. Metapopulation Dynamics: Empirical and Theoretical Investigations. London: Academic Press.

Güémez, J. and M. A. Matías. 1993. Control of chaos in unidimensional maps. Phys. Lett. A 181, 29-32.

Gyllenberg, M., G. Söderbacka and S. Ericsson. 1993. Does migration stabilize local population dynamics? Analysis of a discrete metapopulation model. Math. Biosci. 118, 25-49.

Hassell, M. P. 1975. Density-dependence in single-species models. J. Anim. Ecol. 44, 283-296.

Hassell, M. P., H. N. Comins and R. M. May. 1991. Spatial structure and chaos in insect population dynamics. Nature 353, 255-258. 
Hastings, A. 1993. Complex interactions between dispersal and dynamics: lessons from coupled logistic equations. Ecology 74, 1362-1372.

Hastings, A. and K. Higgins. 1994. Persistence of transients in spatially structured ecological models. Science 263, 1133-1136.

Holt, R. D. and M. P. Hassell. 1993. Environmental heterogeneity and the stability of host-parasitoid interactions. J. Anim. Ecol. 62, 89-100.

Huffaker, C. B. 1958. Experimental studies on predation: dispersion factors and predator-prey oscillations. Hilgardia 27, 343-383.

Levins, R. 1970. Extinction. In Some Mathematical Questions in Biology, M. Gerstenhaber (Ed), pp. 77-107. Providence, RI: American Mathematical Society.

Lima, R. and M. Pettini. 1993. Suppression of chaos by resonant parametric perturbations. Phys. Rev. A 41, 726-733.

Lloyd, A. L. 1995. The coupled Logistic map-a simple model for the effects of spatial heterogeneity on population dynamics. J. Theor. Biol. 173, 217-230.

May, R. M. and G. F. Oster. 1976. Bifurcations and dynamic complexity in simple ecological models. Amer. Nat. 110, 573-599.

Maynard Smith, J. and M. Slatkin. 1973. The stability of predator-prey systems. Ecology 54, 384-391.

McCallum, H. I. 1992. Effects of immigration on chaotic population dynamics. J. Theor. Biol. 154, 277-284.

Ott, E., C. Gregobi and J. A. Yorke. 1990. Controlling chaos. Phys. Rev. Lett. 64, 1196-1199.

Pomeau, Y. and P. Manneville. 1980. Intermittent transition to turbulence in dissipative dynamical systems. Physica A 74, 189-197.

Ruxton, G. D. 1994. Low levels of immigration between chaotic populations can reduce system extinctions by inducing asynchronous regular cycles. Proc. Roy. Soc. London B 256, 189-193.

Schoener, T. 1976. Alternatives to Lotka-Volterra competition: models of intermediate complexity. Theor. Pop. Biol. 10, 309-333.

Sepulchre, J. A. and A. Baboyantz. 1993. Controlling chaos in a network of oscillators. Phys. Rev. E 48, 945-950.

Shinbrot, T., C. Grebogi, E. Ott and J. A. Yorke. 1993. Using small perturbations to control chaos. Nature 363, 411-417.

Solé, R. V. and L. Menéndez de la Prida. 1995. Controlling chaos in discrete neural networks. Phys. Lett. A 199, 65-69.

Stone, L. 1993. Period-doubling reversals and chaos in simple ecological models. Nature 365, $617-620$. 